\title{
A statistical methodology for the comparison of blue gel pen inks analyzed by laser desorption/ionization mass spectrometry
}

\begin{abstract}
A statistical methodology for the objective comparison of LDI-MS mass spectra of blue gel pen inks was evaluated. 33 blue gel pen inks previously studied by RAMAN were analyzed directly on the paper using both positive and negative mode. The obtained mass spectra were first compared using relative areas of selected peaks using the Pearson correlation coefficient and the Euclidean distance. Intra-variability among results from one ink and inter-variability between results from different inks were compared in order to choose a differentiation threshold minimizing the rate of false negative (i.e. avoiding false differentiation of the inks). This yielded a discriminating power of up to $77 \%$ for analysis made in the negative mode. The whole mass spectra were then compared using the same methodology, allowing for a better DP in the negative mode of $92 \%$ using the Pearson correlation on standardized data. The positive mode results generally yielded a lower differential power (DP) than the negative mode due to a higher intra-variability compared to the inter-variability in the mass spectra of the ink samples.
\end{abstract}

Keywords: Questioned document, blue ink, gel pens, mass spectrometry, laser desorption/ionization, discriminating power, Pearson correlation coefficient. 


\section{Introduction}

Ink analysis is an important part of questioned document examination because the presence of several types of inks on a document may be an indication of forgery. In order to distinguish between different ink entries, it is important to have some knowledge of the composition variations in inks available on the market and to select analytical techniques capable of differentiating and classifying these inks. Gel pen inks were first marketed in Japan in the mid 1980s [1,2]. In contrary to traditional aqueous inks containing dyes, gel pen inks contain pigments [3,2], although some of these inks may as well contain dyes [1]. The pigments used often in blue gel pen inks are Pigment blue 15 (i.e. copper phthalocyanine) and Pigment violet 23 (i.e. dioxazine) [1]. The other principal compounds in this type of inks are water, organic solvents soluble in water (i.e. alkylene glycols), natural resins or synthetic polymers, surfactants and additives [2].

Gel pens only recently became widely used compared to other types of writing instruments such as ballpoint pens [3]. This explains the limited research performed in this specific field. Gel pens containing pigments cannot be analyzed by Thin Layer Chromatography (TLC) like dye-based inks [4,5]. Therefore other methods were proposed for the analysis of gel pens inks, such as comparison under different light sources [3]. RAMAN spectroscopy and scanning electron microscopy were then tested and compared $[1,2,6]$, while a preliminary study proposed also $\boldsymbol{\mu}$-XRF spectroscopy [6]. Lately Ion-pairing High Performance Liquid Chromatography (HPLC) and Electrospary tandem Mass Spectrometry (ESI-MS/MS) were used to study the degradation of blue and black gel pen dyes [7,8].

Several studies reported using laser desorption/ionization mass spectrometry (LDI-MS) to analyze other types of inks in a forensic perspective, including the identification of pigments [9-13,5]. LDI-MS has comparable advantages to optical methods, because it requires no sample preparation and it is therefore quick and close to non-destructive. The mass spectra may additionally yield more information about their structure.

The aim of this research was to analyze the inks of 33 blue gel pens by LDI-MS in both positive and negative modes with the aim of differentiating them. In order to evaluate the potential of the technique for comparison of gel pen ink samples, a reliable statistical methodology to compare the mass spectra was developed using the knowledge acquired in other fields of forensic sciences [14-17]. This approach allows an objective comparison limiting the false negative rate (i.e. no false differentiation) using the Pearson correlation coefficient and the Euclidean distance. As the samples were already studied in a previous 
study using Raman spectroscopy [1], the results and discriminating power (DP) of the two methods was also compared.

\section{Experimental}

\section{Samples}

33 blue gel pen inks currently on sale in different countries including Switzerland, Canada, USA, Germany, France and Italy were used in this study. These inks were already analyzed using Raman spectroscopy by Mazzella and Buzzini [1] and all contained pigments (Table 1). The gel pens were used to draw ink lines on white sheets of chlorine-free paper (MIGROS Papeteria $80 \mathrm{~g} / \mathrm{m}^{2}$, Switzerland). For each sample a small piece of paper measuring about $8 \mathrm{x}$ $3 \mathrm{~mm}$ containing the ink line was cut and fixed to a solid steel sample plate using a solventfree glue (UHU ${ }^{\circledR}$ stic, Bühl, Switzerland). Analyses were performed one day later.

\section{LDI-MS}

Mass analyses were carried out on a Bruker Daltonics AutoFlex matrix assisted laserdesorption/ionization reflector time-of-flight (MALDI-TOF) mass spectrometer equipped with a pulsed nitrogen laser $(337 \mathrm{~nm})$. Samples were analysed directly without addition of matrix. Mass spectra of the samples were recorded in positive and negative ion modes. Mass detection was set from 0 to $2000 \mathrm{Da}$. Mass spectra were generated by averaging 50 laser pulses along the ink strokes in positive mode, and 100 laser pulses in negative mode.

The instrument was calibrated using an ink line of a blue ballpoint pen (BIC Cristal) with known composition of dyes determined in previous studies [10,5]. The signals used for calibration in positive mode were the ions at 372.2 and $358.2 \mathrm{~m} / \mathrm{z}$ generated by the dye basic violet 3 (BV3) and the peaks at 456.3 and $428.3 \mathrm{~m} / \mathrm{z}$ of the dye basic violet 4 (BV4). In negative mode the ink of another blue ballpoint pen (Faber-Castell Graf) was used to calibrate the instrument by taking into account the mass signals at 814.0, 734.0 and $654.0 \mathrm{~m} / \mathrm{z}$ produced by the dye solvent blue 38 (SB38).

Signal intensity and peak resolution were used to determine the optimal laser intensities. It was more difficult to find a laser intensity optimal for all the studied gel pens than it was for ballpoint pens in previous studies $[10,18]$. Therefore three laser intensities were selected in both modes (i.e. 30,35 and $40 \%$ for the instrument used in this study) in order to cover the best conditions for all gel pens inks analyzed. This allowed in a first step to study comprehensively the mass spectra of each ballpoint pen in the given optimal laser intensity 
for the specific pen. In a second step, it was then possible to compare the mass spectra from different gel pens using the same analysis conditions (i.e. laser intensity of 35\%). For each of the laser intensities three sets of measurements were performed along the ink lines in positive and negative modes (i.e. per gel pen: 3 replicate analyses x 3 laser intensities x 2 modes $=18$ mass spectra). Blank measurements were performed as well to determine if there was any contribution of the paper or glue interfering with the mass spectra of the ink samples.

\section{Statistical analysis}

The data was treated with PASW statistics 18 (Mathsoft, Inc.), Microsoft Excel (Microsoft Corporation), OriginPro 8.1 (OriginLab Corporation) and R (http://www.r-project-t.org/).

In a first step, identified peaks relative areas (RPAs) were used to discriminate the ink sample. Then additional peaks were selected based on the criteria that their areas were $10 \%$ of the largest signal in the spectra at least in one ink sample. These selected peaks were used as variables for further comparison. Principal Component Analysis (PCA) was carried out to determine the correlation between the selected variables. Finally, the raw data of the mass spectra were extracted in text files and compared directly (corresponding to more than 100 '000 data point per mass spectra covering mass from 0 to $2000 \mathrm{kDa}$ ). For analysis purposes, approximately 60'000 data points were selected as comparison variables in the zone of the spectra where most peaks were found (i.e. from 0 to $750 \mathrm{~m} / \mathrm{z}$ for the positive mode and between 50 and $850 \mathrm{~m} / \mathrm{z}$ for the negative mode).

Pre-treatment methods were applied to reduce the influence of different scales between variables [14-17] : normalization to the sum (within a sample), square root and standardization (subtracting by the mean and dividing by the standard deviation). It should be noted that normalization to the sum does not make sense for all statistical treatments (it is useful for the Euclidean distance, but not for the Pearson correlation). The data was then compared using a Pearson correlation coefficient which was found to be very efficient in previous studies $[14,15]$. The Pearson values range from -1 (anti-correlated) to 1 (correlated). Euclidean was also tested: a value close to 0 indicate a small distance between samples. Calculations yielded two sets of results for each data set: the linked population or intravariability (i.e. correlation values between replicate analyses from the same ink sample) and the unlinked population or inter-variability (i.e. correlation values between ink analyses from different pens). In order to compare the separations obtained between the two populations, 
Receiver Operating Characteristic (ROC) curves were used. A ROC curve is represented by the sensitivity (true negative fraction) as a function of 1-specificity (false positive fraction). The area under the curve quantifies the overlapping degree of the distribution of the two populations. Ideally, if the two distributions do not overlap then a ROC value of 1 is obtained [15-17]. The ROC curves also help to choose a decision threshold (Pearson or Euclidean values) minimizing the false negative or the false positive rates and allow the discriminating power (DP) to be calculated for the given threshold, using the following equation [5]:

$$
D P=1-\frac{2 M}{n(n-1)}
$$

, where $M$ is the number of non-discriminated pairs of samples and $n$ is the total number of samples. The DP indicates the selectivity of the technique used to differentiate the gel pen inks tested.

Distance and correlations coefficient cannot be performed on two variables (i.e., when only two peaks were identified); in that case two inks were considered discriminated when the difference between their mean relative peak areas was superior to their standard variations.

\section{Likelihood ratio calculations}

The obtained Pearson values for comparison of raw spectra were used to estimate the strength of evidence with a likelihood ratio (LR):

$$
\mathrm{LR}=\frac{P\left(E \mid H_{1}\right)}{P\left(E \mid H_{2}\right)}
$$

, where the probability of observing the obtained Pearson values (E) given the ink entries come from the same pen $\left(\mathrm{H}_{1}\right)$ is compared to the probability of observing the same Pearson values $(\mathrm{E})$ given the ink entries come from different pens $\left(\mathrm{H}_{2}\right)$.

Based on a previous publication [19], Kernel Density Estimation (KDE) with a Gaussian kernel for each data point (i.e., distance measurement) was used. The LR was obtained by the density of correlation (d) between two measurements $\mathrm{x}$ and $\mathrm{y}$ given the two alternative hypotheses $\mathrm{H}_{1}$ and $\mathrm{H}_{2}$ [19]:

$$
\mathrm{LR}=\frac{f\left(\delta(\mathbf{x}, \mathbf{y}) \mid H_{p}\right)}{f\left(\delta(\mathbf{x}, \mathbf{y}) \mid H_{d}\right)}=\frac{f\left(\partial\left(\left\{x_{d}, x_{t h}, x_{w}\right\},\left\{y_{d}, y_{t h}, y_{w}\right\}\right) \mid H_{p}\right)}{f\left(\partial\left(\left\{x_{d}, x_{t h}, x_{w}\right\},\left\{y_{d}, y_{t h}, y_{w}\right\}\right) \mid H_{d}\right)}
$$

, where $\mathrm{f}$ is the Kernel density function. 
The obtained data was divided in three groups for this purpose:

- Reference data $X$ representing measurements from a reference sample with which other samples were compared. $\mathrm{X}$ was constituted of the random half of the correlation values between replicate analyses from the same ink sample $(n=50)$

- Comparison data $Y$ representing another sample which was compared with the reference sample $\mathrm{X}$ and the population data Z. For research purposes, two types of Y samples were created: same ink data using the other random half of the correlation values between replicate analyses from the same ink sample $(n=49)$ and randomly selected half of the different inks correlation values $(n=264)$.

- Population data $Z$ representing the other random half of the correlation values between different ink samples $(\mathrm{n}=264)$.

\section{Results and discussion}

\section{Identified peaks}

In all gel pen inks, the molecular ions at $575.1 \mathrm{~m} / \mathrm{z}$ (in positive and negative modes) and at $588.1 \mathrm{~m} / \mathrm{z}$ (in positive mode only) were detected. Their isotopic distribution indicated the presence of pigment blue 15 (PB15) and pigment violet 23 (PV23) respectively (Figure 1).

These two pigments were already identified in those gels pen inks using RAMAN [1], but while PB15 was identified in all the investigated inks, PV23 was recorded only in $55 \%$ of them. LDI-MS was thus more sensitive to detect PV23. The relative peak area of PV23 to PB15 ranged from 1 to $25 \%$. This indicated that the peak area of PV23 was always significantly lower in comparison to the peak area of PB15. Using the relative peak area of these two signals to differentiate the inks yielded a discriminating power (DP) of $72 \%$. This value is only slightly better than the one obtained previously by RAMAN (i.e. PD=68\%) [1].

\section{Selected peaks}

Many additional peaks were recorded in the mass spectra but could not be attributed to known pigments or molecules. Using only the identified peaks mean that significant information 
might be lost for efficient comparison. Therefore peak areas of additional peaks were compared in a second step in order to improve the DP of the LDI-MS method. The peaks were selected on the basis of their relative peak (RPA) area to PB15 in the mass spectra acquired with a laser intensity of $30 \%$, i.e. if their RPA to PB15 was above $10 \%$ in the mass spectra of at least one ink, the given $\mathrm{m} / \mathrm{z}$ was chosen as a comparison variable (VAR). This yielded 15 VARs in the positive mode and 10 VARs in the negative mode including $\mathrm{m} / \mathrm{z}=575.1$ in both modes (see Table 2 ).

The peak areas were compared using the Pearson correlation coefficient or Euclidean distance. Replicate analyses from the same sample were first compared (i.e. INTRAvariability, 99 comparisons) and then analyses from different samples were also compared (i.e. INTER-variability, 528 comparisons). This data was used to plot a histogram of the two populations (example in Figure 5 - above). Performing then a ROC analysis on that data allowed evaluating the overlapping area of the two distributions (example in Figure 5 below): the closer to 1 the area under the ROC curve, the better the separation. To diminish the influence of large variable such as PB15 (VAR10 and VAR8 in the positive and negative mode respectively) two pre-treatments were applied: square root and standardization of a given VAR. Normalization of the data to the sum of all variables in a sample was additionally performed for Euclidean calculation in order to diminish the variations from one analysis to another. These pre-treatments allowed a significant improvement in the separation of the distribution in both the negative and positive mode (Table 3). While square root followed by Pearson was the best pre-treatment for the negative mode (area under the ROC curve of 0.974), standardization followed by Pearson was the best in positive mode (area under the ROC curve of 0.958 ). Results showed globally a better separation for the negative mode and Pearson was found to be more efficient than Euclidean for separation of the distributions as was expected given previous literature [15].

While in some field of forensic sciences the aim is to minimize the number of false positives [14-17], the objective in the comparison of ink samples is more to minimize the number of false negatives, i.e. avoiding a false differentiation of questioned ink samples. From the data of the ROC curves, it was possible to extrapolate the DP (or the specificity of the method) for a $0 \%$ false negative rate (or 1 -sensitivity) (Tables 4 and 5 for positive and negative mode respectively). For example, in the positive mode a Pearson value of 0.149 was obtained for the overlapping area with a DP of $63 \%$ (Table 4). Two inks were therefore considered discriminated when the obtained Pearson values for their comparison were under 0.149. This chosen threshold yielded no false discrimination. However this also meant that $37 \%$ false 
positives were recorded (i.e. different samples that were not differentiated). When aiming at a minimum false positive rate however, a Pearson threshold value of 0.995 was obtained for a DP of 100\% (Table 4). With this threshold, two samples would be considered discriminated if the obtained comparison Pearson values were under 0.995. However this would also mean a $95 \%$ false negative rate (i.e. same samples that would be wrongly differentiated). The data from the ROC curves (summarized in Table 4 - 5) help therefore selecting the adequate Pearson decision threshold for a given purpose, which is here differentiation of inks avoiding false negative. In the negative mode a higher DP was yielded (75\%) because the Pearson threshold value for a 0\% false negative rate reached 0.507 (Table 5). Regarding Euclidean distances, it was not possible to minimize the false negative rate to $0 \%$ and still differentiate inks. Therefore this method was not adapted for the treatment of LDI-MS mass spectra of blue gel pen inks.

Combining the information yielded by the two modes was interesting as it was to some extent complementary. In fact for a $0 \%$ false negative rate, 41 pairs of samples differentiated in the positive mode were not differentiated in the negative mode, while 97 pairs of samples were only differentiated in the negative mode. This meant that a total of 431 pairs could be differentiated when combining the results obtained in the two analysis mode for a total DP of 0.82 (Table 6). For example comparison of the pair of samples 31 and 33 (Figure 2) yielded a Pearson value above the differentiation threshold (i.e. $0.383>0.149$ in Table 4) and was thus not differentiated in the positive mode, while the Pearson value obtained in the negative mode allowed differentiation of the samples (i.e. $0.176<0.527$ in Table 5).

To evaluate the pertinence of the chosen variables, their correlation was evaluated using Principal Component Analysis (PCA) (Figure 3): if the variables were overlapping or close to each other, they were correlated. In positive mode, the following variables were correlated: VAR2 and 3; VAR4 and 5 (Figure 4 - right); VAR7, 8, 9 and 11; VAR12 and 13 (Figure 4 left); VAR10 and 15. In the negative mode, only the VAR 3, 4, 5 and 8 were correlated together. These results were then confirmed with correlation coefficients. A value above 0.9 was obtained among these groups of variables.

Moreover it was also deducted from the PCA which variables were the most efficient for the differentiation of the ink samples. If the variables were close to the interior of the circle, they were not very pertinent for the differentiation. For example, VAR 4 and 5 were more variable among the different ink samples, than VAR 12 and 13 (Figure 3). The latter essentially differentiated sample 11 from the other inks. 
This information was used to test different combinations without the correlated variables for comparison of the samples (Table 6). This allowed increasing the DP up to 69\% for a comparison with twelve variables in the positive mode (excluding VAR2, 5 and 12). In some configurations the DP also decreased significantly, thus showing that VAR10 and 15 were both useful. In the negative mode, excluding VAR 4, 5 and 8 allowed increasing the DP to $77 \%$. These results seem to indicate that the intra-variability of the excluded variables of one sample was sometimes more significant than the inter-variability between samples. This confirmed the fact that the choice of variables is an essential step for the comparison of ink samples and should not be underestimated [17].

\section{Raw mass spectrum data}

Much more information was recorded in the mass spectra (more than $600^{\prime} 000 \mathrm{~m} / \mathrm{z}$ values per mass spectrum). Therefore a direct statistical comparison of the whole data set was attempted using Pearson coefficient and ROC curves. In a first step, the spectra acquired in the three laser intensities were respectively compared for the two analysis modes (Table 7). The best separation of the INTRA and INTER-variability distributions was obtained this time for spectra in the positive mode with a laser intensity of $40 \%$ (Figure 5). The areas under the ROC curve were generally lower for the negative mode spectra, with the best separation for spectra acquired with a laser intensity of $35 \%$.

In a second step, the raw data giving the best separation for each mode was selected for further statistical analysis. Two pre-treatments were tested to diminish the influence of large peaks in the mass spectra such as PB15: square root and standardization of $\mathrm{m} / \mathrm{z}$. The best separation was then obtained for the standardized data acquired in the negative mode (ROC area $=0.986$ ), followed by the data pre-treated with the square root acquired in the positive mode $($ ROC area $=0.938)$, very close to the standardized data $($ ROC area $=0.936)($ Table 8$)$. It is interesting to note that the DP for a $0 \%$ false negative rate was very low for the standardized data acquired in positive mode (laser intensity of 40\%). A Pearson threshold value of -0.04 was obtained for the overlapping area. This indicated a poor intra-variability and yielded thus a low DP of $45 \%$. In the negative mode the results were on the contrary very promising, as the threshold value for $0 \%$ false negative reached 0.19 for a DP of $92 \%$. These results indicated a much better intra-variability among same ink samples in the negative mode compared to the positive mode. Combining the two sets of data did not allow additional discrimination, because only one complementary pair was differentiated for the positive mode in comparison to the negative mode, thus increasing the total DP of only $0.2 \%$ (Table 9). 


\section{Likelihood ratio}

The LR obtained using Pearson coefficient values from comparison of raw spectrum data varied a lot within comparisons of the same ink (i.e. $\mathrm{H}_{1}$ is true) as well as between comparisons of different inks (i.e. $\mathrm{H}_{2}$ is true). In the positive mode, the median of LR values reached 10 for $\mathrm{H}_{1}$ true and 0.24 for $\mathrm{H}_{2}$ is true (Figure $6-$ left). In the negative mode, the median values were slightly better with 19 for $\mathrm{H}_{1}$ true and 0.0007 for $\mathrm{H}_{2}$ is true (Figure $6-$ right). However the minimal and maximal values obtained for both hypotheses overlap largely, particularly in the positive mode. These results confirm that negative mode LDI-MS is more adequate to discriminate inks (Figure 6 - right - red box).

As demonstrated earlier [19], the strength of evidence of Pearson coefficients (i.e. all variables reduced to one value) is generally lower than for a multivariate approach. Moreover gel pen inks are mass products distributed largely around the world; it is therefore difficult to collect representative data in order to interpret the significance of the ink analysis results. A non-differentiation is particularly difficult to interpret (black boxes in Figure 6; e.g. one can conclude that two ink entries are of the same chemical composition, however this does not mean they were drawn by the same pen). It is more straightforward to conclude that two inks are different (red boxes in Figure 6; e.g. they come from different pens).

\section{Conclusion}

The proposed methodology enabled an objective comparison of LDI-MS mass spectra from the 33 blue gel pen inks through statistical treatments. Pearson correlation coefficient following standardization or square root was found to be very efficient to distinguish between the inks, most particularly for the negative mode results. Additionally to being objective, this approach did include the intra-variability as part of the procedure and thus, insured that no false differentiation occurred, i.e. the decision threshold was fixed to obtain a $0 \%$ false negative rate. When comparing relative peak areas, the selection of the variables was important: they should ideally be very variables between samples, while being reproducible between samples. Refined selection of the variables allowed to reach a maximal DP in the positive mode of $69 \%$ which was very similar to the one obtained previously by RAMAN for the same ballpoint pen inks, and a DP of $77 \%$ in the negative mode. The positive mode gave generally lower DP, probably due to a high variability within results from a same sample 
(intra-variability) in comparison to the inter-variability between samples. The best DP of 92\% was obtained by comparison of the whole mass spectra acquired in the negative mode. Only 41 pairs of samples for a total of 528 remained thus undifferentiated. The proposed statistical approach can be applied to the data obtained by other analytical method, allowing more objectivity, quicker comparison of the data and fewer false negatives. The robustness of the methodology should further be evaluated by comparing obtained decision threshold among different LDI-MS instrumentation.

\section{Acknowledgment}

The authors wish to thank Prof. Pierre Esseiva for his useful advice on the statistical treatments of the data. This work has been kindly supported by Swiss National Science Foundation (Fund No. PP00P1_123358/1).

\section{References}

[1] W. Mazzella, P. Buzzini, Raman spectroscopy of blue gel pen inks. Forensic Science International 152 (2005) 241-247.

[2] W. Mazzella, A. Khanmy-Vital, A Study to Investigate the Evidential Value of Blue Gel Pen Inks. Journal of Forensic Sciences 48 (2) (2003) 1-6.

[3] M.N. Gernandt, J.J. Urlaub, An Introduction to the Gel Pen. Journal of Forensic Sciences 41 (3) (1996) 503-504.

[4] J.A. Tappolet, The high-performance thin layer chromatography (HPTLC) - its application to the examination of writing inks. Forensic Science International 22 (1983) 99-109.

[5] C. Weyermann, R. Marquis, W. Mazzella, B. Spengler, Differentiation of Blue Ballpoint Pen Inks by Laser Desorption Ionization Mass Spectrometry and High-Performance Thin-Layer Chromatography. Journal of Forensic Sciences 52 (1) (2007) 216-218.

[6] J. Zieba-Palus, R. Borusiewicz, M. Kunicki, PRAXIS-combined $\mu$-Raman and $\mu$-XRF spectrometers in the examination of forensic samples. Forensic Science International 175 (2008) 1-10.

[7] Y.-Z. Liu, J. Yu, M.-X. Xie, Y. Chen, G.-Y. Jiang, Y. Gao, Studies on the degradation of blue gel pen dyes by ion-pairing high performance liquid chromatography and electrospray tandem mass spectrometry. Journal of Chromatography A 1125 (2006) 95-103.

[8] Y.-Z. Liu, J. Yu, M.-X. Xie, Y. Liu, J. Han, T.-T. Jing, Classification and dating of black gel pen ink by ion-pairing high-performance liquid chromatography. Journal of Chromatography A 1135 (2006) 56-57.

[9] K. Papson, S. Stachura, L. Boralsky, J. Allison, Identification of Colorants in Pigmented Pen Inks by Laser Desorption Mass Spectrometry. Journal of Forensic Sciences 53 (1) (2008) 100106.

[10] M. Gallidabino, C. Weyermann, R. Marquis, Differentiation of blue ballpoint pen inks by positive and negative mode LDI-MS. Forensic Science International on-line (2010).

[11] D. Grim, J. Allison, J.A. Siegel, Determining the Age of an Ink on a Document in Question Using Laser Desorption / Ionization Mass Spectrometry, American Academy of Forensic Sciences, Seattle, 2001. 
[12] D.M. Grim, J. Allison, Identification of colorants as used in watercolor and oil paintings by UV laser desorption mass spectrometry. International Journal of Mass Spectrometry 222 (2003) 85-99.

[13] D.M. Grim, J.A. Siegel, J. Allison, Evaluation of laser desorption-ionization mass spectrometry methods applications of the analysis of inks on paper. Journal of Forensic Sciences 46 (6) (2002) 1411-1420.

[14] P. Esseiva, L. Dujourdy, F. Anglada, F. Taroni, P. Margot, A methodology for illicit heroin seizures comparison in a drug intelligence perspective using large databases. Forensic Science International 132 (2003) 139-152.

[15] S. Lociciro, P. Esseiva, P. Hayoz, L. Dujourdy, F. Besacier, P. Margot, Cocaine profiling for strategic intelligence, a cross-border project between France and Switzerland. Part II. Validation of the statistical methodology for the profiling of cocaine. Forensic Science International 177 (2008) 199-206.

[16] R. Marquis, C. Weyermann, C. Delaporte, P. Esseiva, L. Dujourdy, C. Koper, L. Aalberg, R. Dahlenburg, F. Zrcek, J. Bosenko, Drug intelligence based on MDMA tablets data: (2) Physical characteristics profiling. Forensic Science International 178 (1) (2008) 24-39.

[17] C. Weyermann, R. Marquis, C. Delaporte, P. Esseiva, L. Dujourdy, E. Lock, L. Aalberg, S. Dieckmann, F. Zrcek, J. Bosenko, Drug intelligence based on MDMA tablets data: (1) Organic impurities profiling. Forensic Science International 177 (1) (2008) 11-16.

[18] C. Weyermann, D. Kirsch, C. Costa-Vera, B. Spengler, Photofading of Ballpoint Dyes Studied on Paper by LDI and MALDI MS. Journal of the American Society for Mass Spectrometry 17 (3) (2006) 297-306.

[19] A. Bolck, C. Weyermann, L. Dujourdy, E. P., J. van den Berg, Different likelihood ratio approaches to evaluate the strength of evidence of MDMA tablet comparisons. Forensic Science International 191 (2009) 42-51. 


\begin{tabular}{|c|c|}
\hline $\mathbf{N}^{\circ}$ & Brand \\
\hline 1 & BIC Intensity (CAN) \\
\hline 2 & BIC Intensity $(\mathrm{CH})$ \\
\hline 3 & Brio Scatto Gel \\
\hline 4 & Edding 2170 \\
\hline 5 & Edding 2189 \\
\hline 6 & Flair Jel Tech 250 \\
\hline 7 & Focus II \\
\hline 8 & Focus LX \\
\hline 9 & Herlitz Zebra \\
\hline 10 & Marvel Gel Excel \\
\hline 11 & Merangue Hi-jell \\
\hline 12 & Mondial LUS \\
\hline 13 & Montex HY-Power \\
\hline 14 & Papermate Gel Glide \\
\hline 15 & Pentel K106 \\
\hline 16 & Pentel K118 \\
\hline 17 & Pentel K160 \\
\hline 18 & Pentel K227 (CH) \\
\hline 19 & Pentel K227 (USA) \\
\hline 20 & Pentel KN127 \\
\hline 21 & Pilot G-Tec C4 \\
\hline 22 & Pilot P-500 \\
\hline 23 & Sakura MED \\
\hline 24 & Sakura XPGB (USA) \\
\hline \multirow{2}{*}{25} & Sanford Uniball Gel \\
\hline & Grip \\
\hline 26 & Schneider Gel-TOPS \\
\hline 27 & Staples \\
\hline 28 & Uniball Signo UM-133 \\
\hline 29 & Uniball Signo UM-152 \\
\hline 30 & Uniball Signo UM-153 \\
\hline 31 & Zebra Antique \\
\hline 32 & Zebra Jimnie Gel \\
\hline 33 & Zebra Sarasa \\
\hline
\end{tabular}

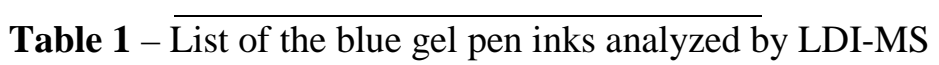




\begin{tabular}{|c|c|c|c|c|}
\hline \multirow{2}{*}{ Variable \# } & \multicolumn{2}{|c|}{ Positive mode } & \multicolumn{2}{|c|}{ Negative mode } \\
\hline & $\mathbf{m} / \mathbf{z}$ & $\begin{array}{c}\text { Isotopic } \\
\text { distribution }\end{array}$ & $\mathbf{m} / \mathbf{z}$ & $\begin{array}{c}\text { Isotopic } \\
\text { distribution }\end{array}$ \\
\hline VAR 1 & 131.5 & & 145.5 & \\
\hline VAR 2 & 228.0 & & 252.6 & \\
\hline VAR 3 & 382.6 & & 269.8 & \\
\hline VAR 4 & 410.6 & & 412.9 & \\
\hline VAR 5 & 438.6 & & 431.0 & \\
\hline VAR 6 & 478.3 & & 495.8 & \\
\hline VAR 7 & 485.5 & & 529.8 & \\
\hline
\end{tabular}


VAR 8

520.2

VAR 9

VAR 10

VAR 11

VAR 12

VAR 13

VAR 14

VAR 15

560.2

575.1

588.1

652.6

733.5

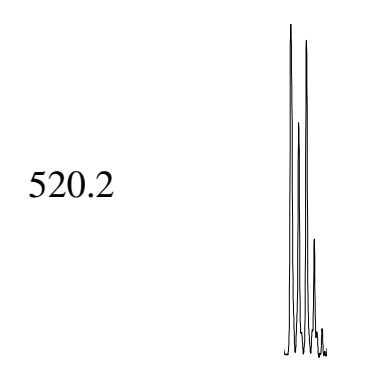

575.1
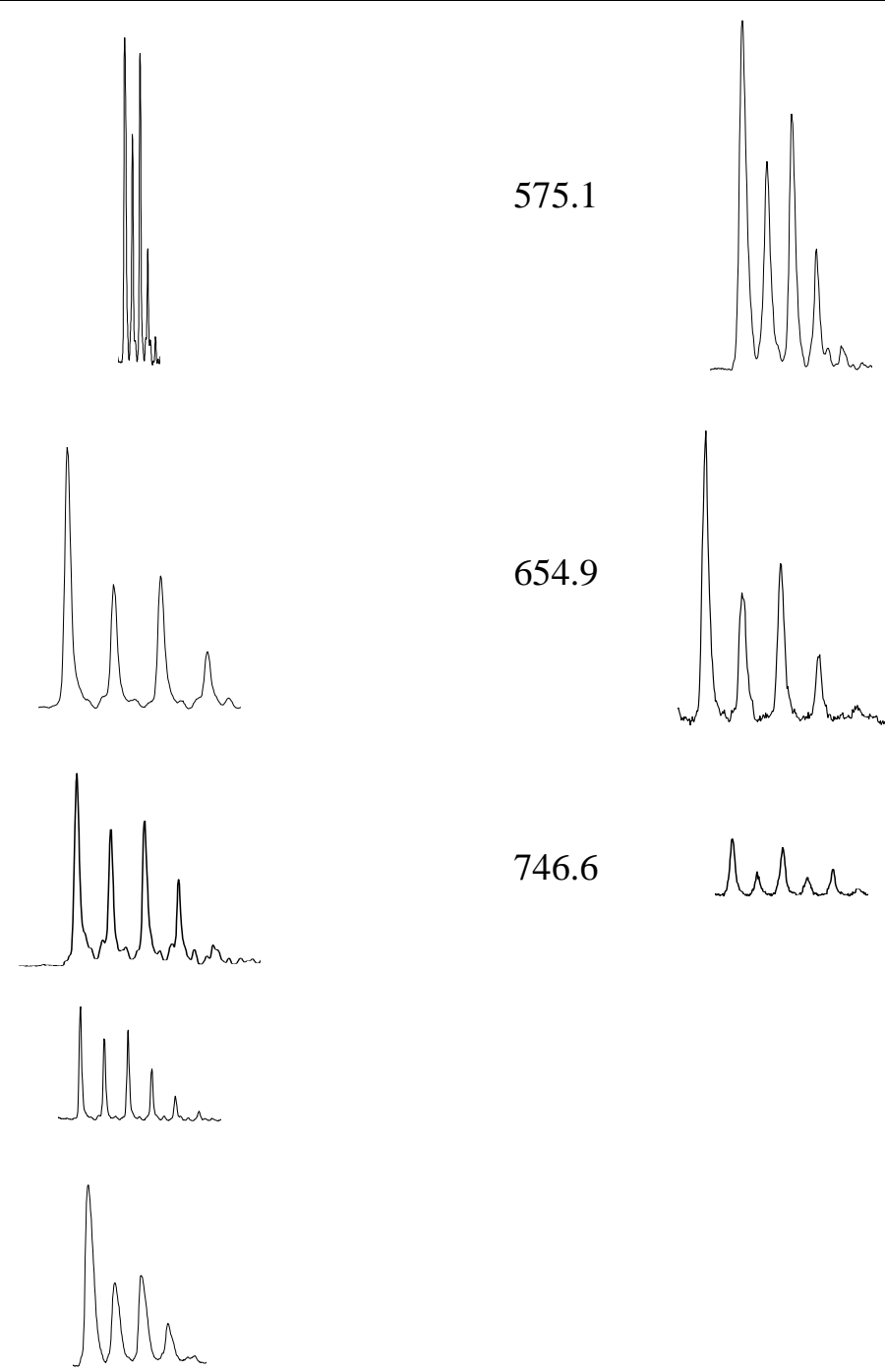

686.5
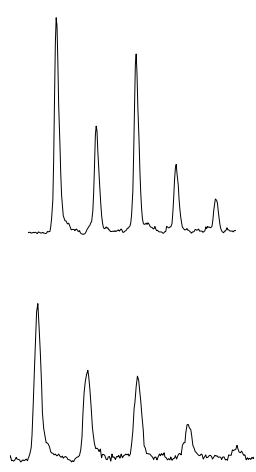

654.9

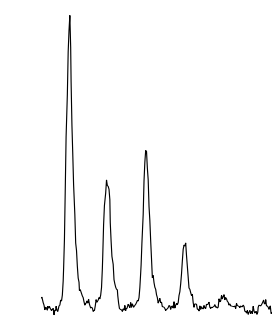

746.6

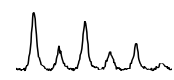

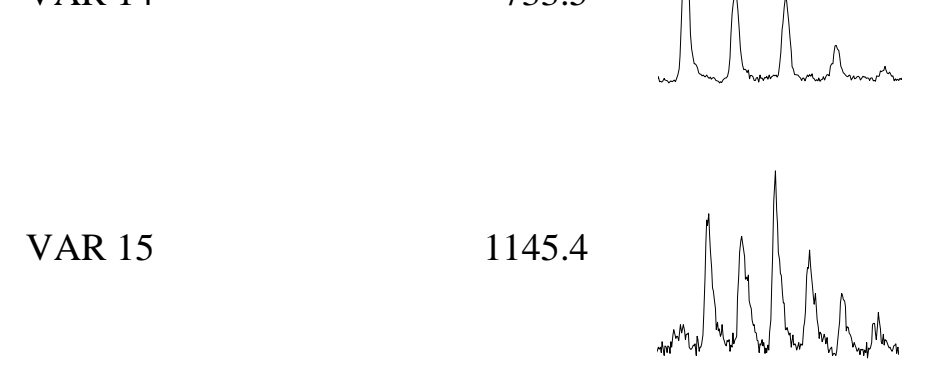

Table 2 - Selected variables for the statistical comparison of blue gel pen inks. The peak areas of the variables were used for comparison. 


\begin{tabular}{ccccc}
\hline RPA & \multicolumn{2}{c}{ Positive mode } & \multicolumn{2}{c}{ Negative mode } \\
& Pearson & Euclidean & Pearson & Euclidean \\
\hline raw & 0.897 & 0.780 & 0.960 & 0.734 \\
normalization to the sum (sample) & 0.897 & 0.928 & 0.960 & 0.846 \\
square root & 0.943 & 0.897 & 0.974 & 0.773 \\
$\begin{array}{c}\text { normalization to the sum + square } \\
\text { root }\end{array}$ & 0.943 & 0.953 & 0.974 & 0.859 \\
standardization (m/z) & $\mathbf{0 . 9 5 8}$ & 0.832 & 0.954 & 0.743 \\
\hline
\end{tabular}

Table 3 - Area under the ROC curves for the comparison of mass spectra. The best separations are highlighted for the positive and negative mode (bold).

\begin{tabular}{ccccc}
\hline $\begin{array}{c}\text { Pearson } \\
\text { threshold }\end{array}$ & $\begin{array}{c}\text { False Negative } \\
\text { (1-sensitivity) }\end{array}$ & $\begin{array}{c}\text { False Positive } \\
\text { (1-specificity) }\end{array}$ & $\begin{array}{c}\text { Number of } \\
\text { differentiated } \\
\text { pairs }\end{array}$ & DP \\
\hline 0.149 & $0 \%$ & $37 \%$ & 334 & $\mathbf{0 . 6 3}$ \\
0.361 & $2 \%$ & $21 \%$ & 418 & 0.79 \\
0.573 & $14 \%$ & $10 \%$ & 474 & 0.90 \\
0.784 & $26 \%$ & $5 \%$ & 505 & 0.96 \\
0.995 & $95 \%$ & $0 \%$ & 528 & 1.00 \\
\hline
\end{tabular}

Table 4 - Values extrapolated from the ROC curves on standardised data acquired in positive mode. The DP allowing 0 false negative is $65 \%$ (bold).

\begin{tabular}{ccccc}
\hline Pearson threshold & $\begin{array}{c}\text { False Negative } \\
\text { (1-sensitivity) }\end{array}$ & $\begin{array}{c}\text { False Positive } \\
\text { (1-specificity) }\end{array}$ & $\begin{array}{c}\text { Number of } \\
\text { differentiated } \\
\text { pairs }\end{array}$ & DP \\
\hline 0.5068 & $0 \%$ & $25 \%$ & 390 & $\mathbf{0 . 7 5}$ \\
0.6126 & $3 \%$ & $19 \%$ & 418 & 0.82 \\
0.7325 & $11 \%$ & $14 \%$ & 440 & 0.86 \\
0.8509 & $18 \%$ & $8 \%$ & 485 & 0.92 \\
0.9975 & $95 \%$ & $0 \%$ & 528 & 1.00 \\
\hline
\end{tabular}

Table 5 - Values extrapolated from the ROC curves on standardised data acquired in negative mode. The DP allowing 0 false negative is $75 \%$ (bold). 


\begin{tabular}{|c|c|c|c|c|}
\hline & \# of variables & $\begin{array}{l}\text { Variables } \\
\text { excluded }\end{array}$ & $\begin{array}{c}\text { Number of } \\
\text { differentiated pairs }\end{array}$ & $\begin{array}{c}\text { DP } \\
(0 \% \text { false negative })\end{array}$ \\
\hline \multirow{8}{*}{ Positive mode } & 15 & - & 334 & 0.63 \\
\hline & 14 & VAR12 & 348 & 0.66 \\
\hline & 14 & VAR15 & 290 & 0.55 \\
\hline & 14 & VAR2 & 354 & 0.67 \\
\hline & 14 & VAR5 & 338 & 0.64 \\
\hline & 12 & VAR7,8,9 & 317 & 0.60 \\
\hline & 12 & VAR2,5,12 & 364 & 0.69 \\
\hline & 8 & VAR2,5,7,8,9,12,15 & 290 & 0.55 \\
\hline \multirow{2}{*}{ Negative mode } & 10 & - & 390 & 0.75 \\
\hline & 7 & VAR $4,5,8$ & 407 & 0.77 \\
\hline Combined & 23 & - & 431 & 0.82 \\
\hline
\end{tabular}

Table 6 - Values extrapolated from the ROC curves on selected standardised relative peak areas. The best DP allowing 0 false negative is above $81 \%$ when results from both analysis modes were combined (bold).

\begin{tabular}{ccc}
\hline ROC area & Positive mode & Negative mode \\
\hline Laser intensity 30\% & 0.862 & 0.809 \\
Laser intensity 35\% & 0.891 & 0.838 \\
Laser intensity 40\% & $\mathbf{0 . 9 1 9}$ & 0.832
\end{tabular}

Table 7 - Area under the ROC curves for the comparison of mass spectra. The best separation is obtained for spectra acquired in positive mode with a laser intensity of $40 \%$ (bold). 


\begin{tabular}{ccc}
\hline ROC area & $\begin{array}{c}\text { Positive mode } \\
\text { Laser intensity 40\% }\end{array}$ & $\begin{array}{c}\text { Negative mode } \\
\text { Laser intensity 35\% }\end{array}$ \\
\hline raw & 0.919 & 0.838 \\
square root & 0.938 & 0.904 \\
Standardization & & $\mathbf{0 . 9 8 6}$ \\
(per variable) & 0.936 & \\
\hline
\end{tabular}

Table 8 - Area under the ROC curves for the comparison of mass spectra. The best separation is obtained for spectra acquired in positive mode with a laser intensity of $40 \%$ (bold).

\begin{tabular}{|c|c|c|}
\hline Raw data & \# differentiated pairs & DP \\
\hline Positive mode & 238 & 0.4508 \\
\hline Negative mode & 486 & 0.9204 \\
\hline Combined mode & 487 & $\mathbf{0 . 9 2 2 3}$ \\
\hline
\end{tabular}

Table 9 - Number of pairs differentiated and discriminating power (DP) in function of the analysis mode for standardized raw data (laser intensity of $40 \%$ and $35 \%$ in positive and negative mode respectively). The best DP was obtained when both modes were combined (bold). 


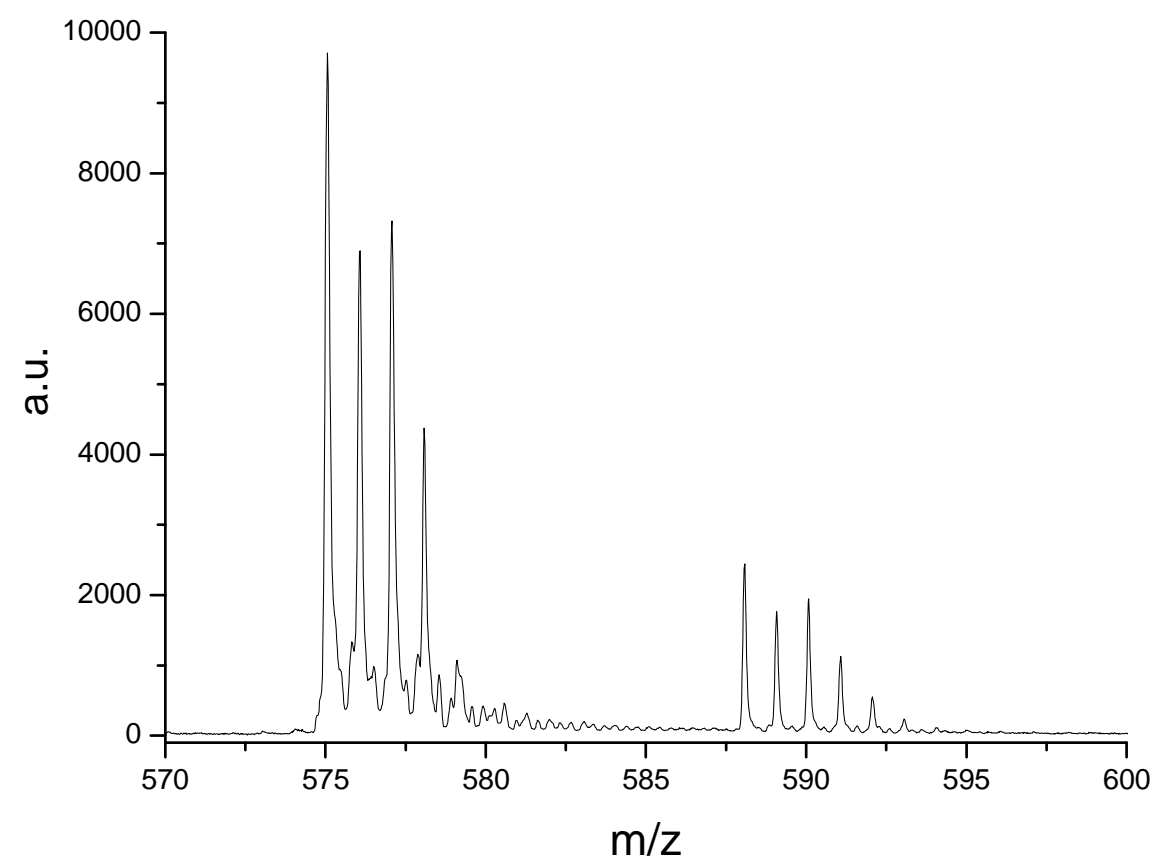

Figure 1 - Isotopic distributions of pigment blue $15(\mathrm{~PB} 15$; m.i. $=575.1 \mathrm{~g} / \mathrm{mol})$ and pigment violet $23(\mathrm{PV} 23 ; \mathrm{m} . \mathrm{i} .=588.1 \mathrm{~g} / \mathrm{mol})$ in the ink from ballpoint pen 32. 


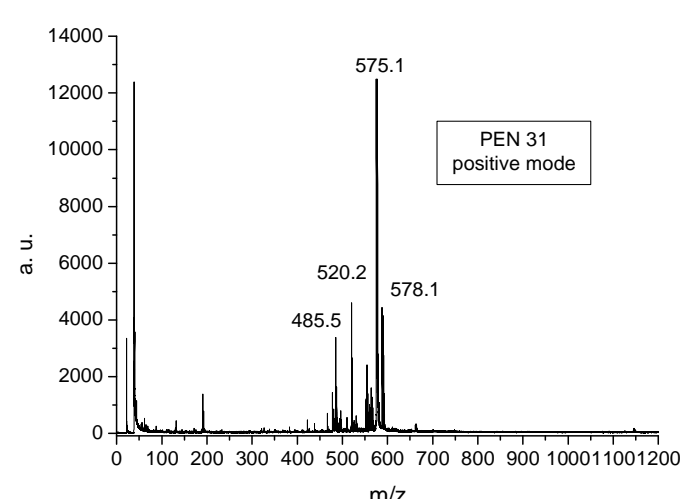

$\mathrm{m} / \mathrm{z}$

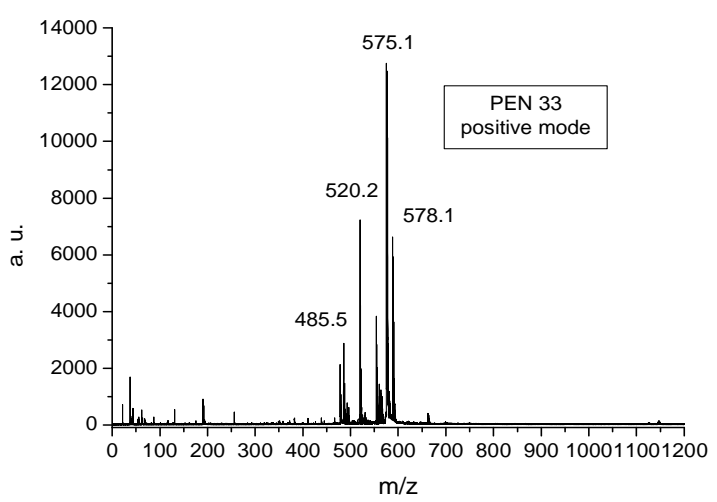

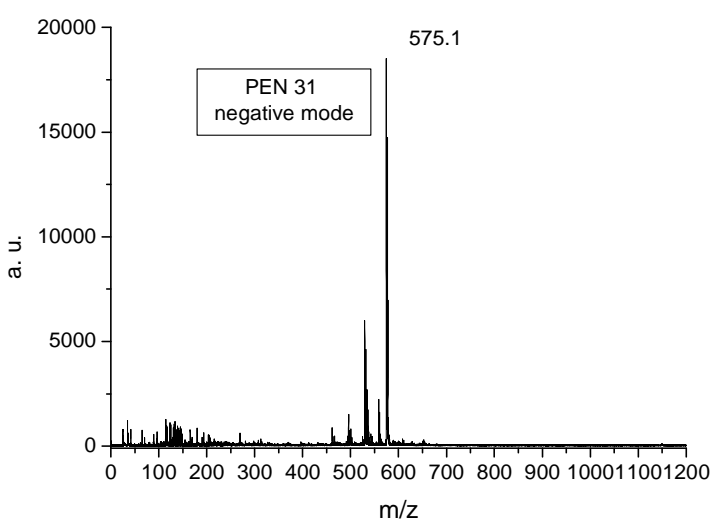

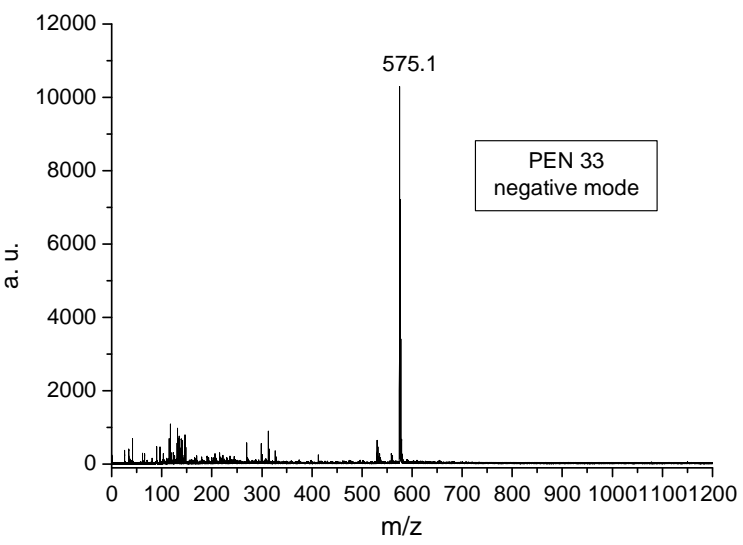

Figure 2 - LDI-MS mass spectra of PEN 31 and PEN 33 acquired in the positive mode (right) and negative mode (left). This pairs of samples was not discriminated in the positive mode, but was in the negative mode. 

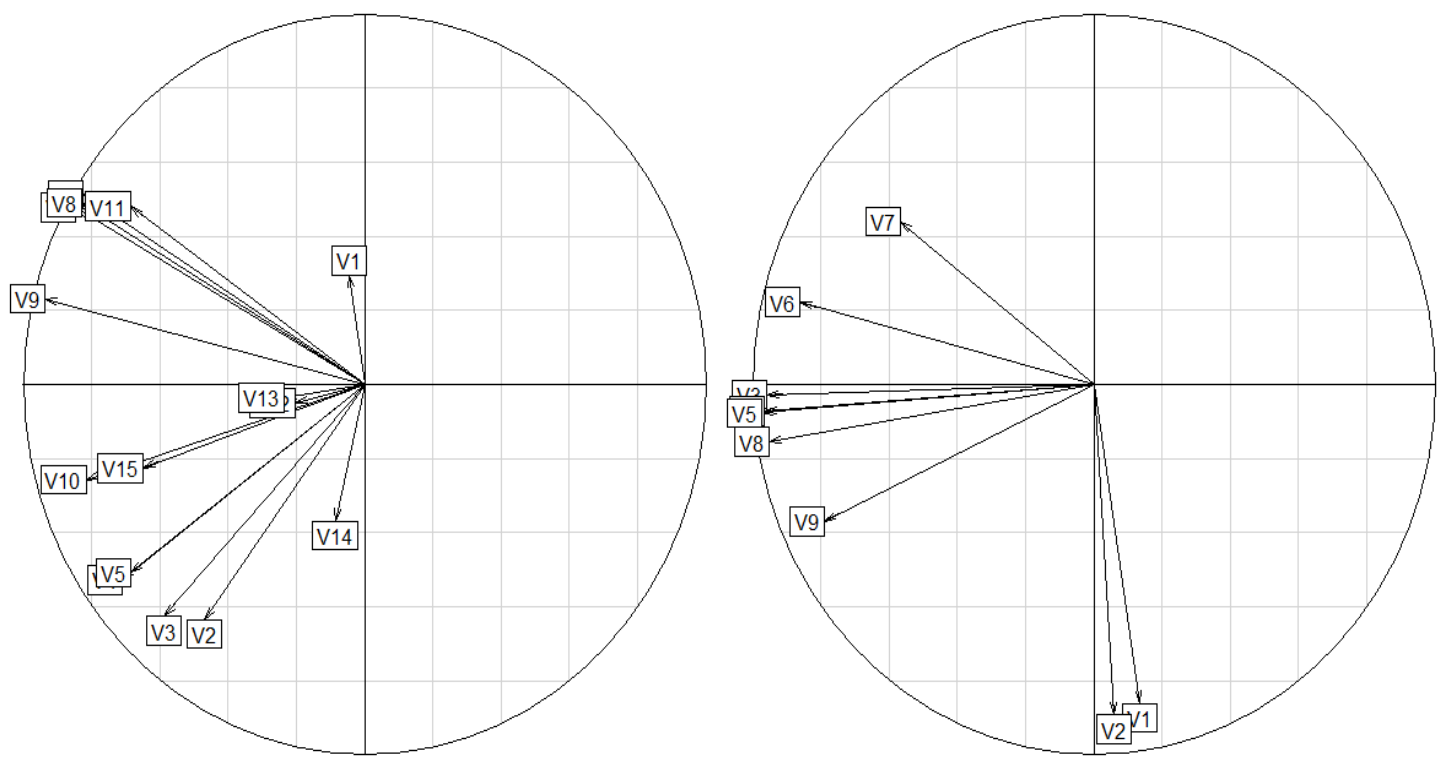

Figure 3 - PCA of the variables (VAR) used for classification of the gel pen inks samples for mass spectra acquired in the positive mode (right) and negative mode (left). 

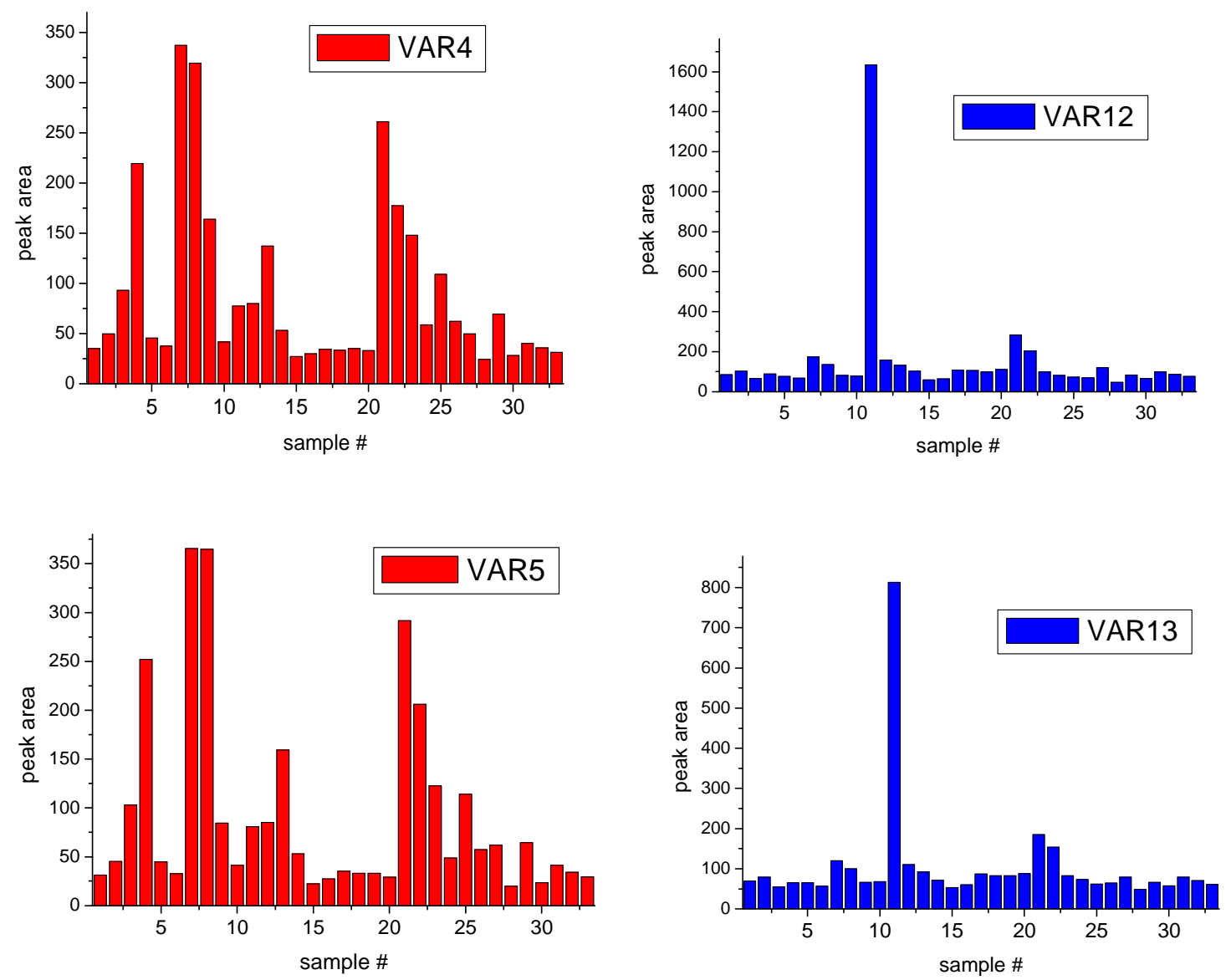

Figure 4 - LEFT: Distribution of VAR4 $(382 \mathrm{~m} / \mathrm{z})$ and VAR5 $(410 \mathrm{~m} / \mathrm{z})$ among the 33 samples. These two variables are correlated (correlation coefficient of 0.9815 ), but very variables between samples. RIGHT: Distribution of VAR12 $(652 \mathrm{~m} / \mathrm{z})$ and VAR13 $(686 \mathrm{~m} / \mathrm{z})$ among the $33 \mathrm{samples}$. These two variables are also correlated (correlation coefficient of 0.9986), but as can be seen, they are essentially efficient to differentiate sample 11 from the others. 

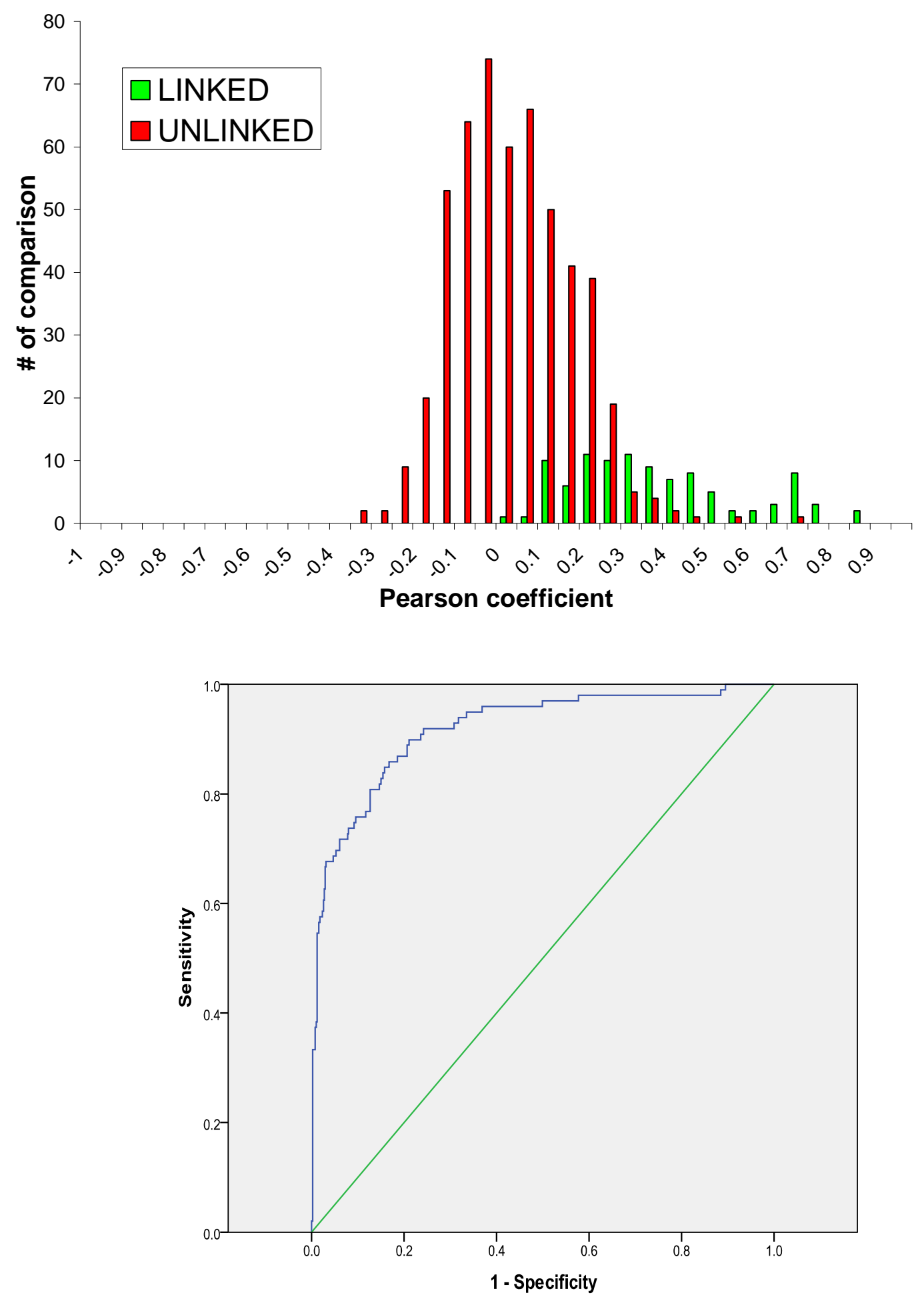

Figure 5 - Above: Histogram representing Pearson correlation coefficient between distribution of mass spectra acquired in positive mode with a laser irradiance of $40 \%$ from a same pen (INTRA) and from different pens (INTER). Below: ROC curve of the overlapping area of the two distributions represented above (Pearson values from -0.04 to 0.68) yielding an area under the curve of 0.919 . 

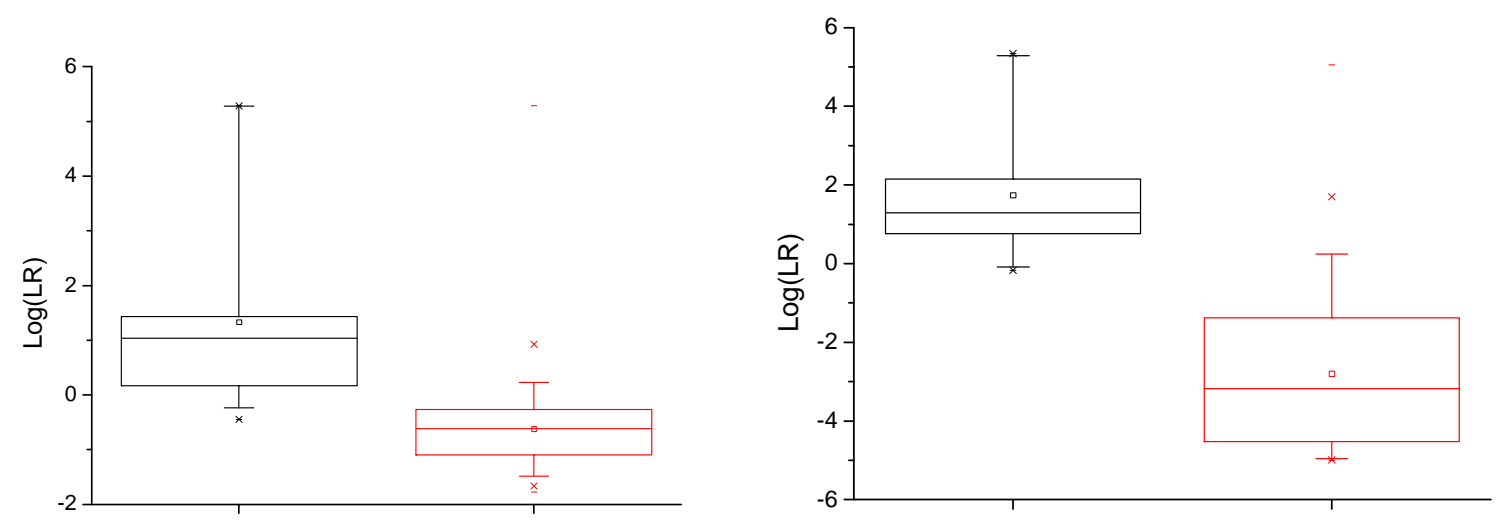

Figure 6 - Boxplots representing the LRs calculated for comparison of inks by LDI-MS in positive mode (Left) and negative mode (right). The calculations are based on Pearson coefficients calculated between raw data from the spectra of the inks. In black, values obtained for same ink samples were compared $\left(\mathrm{H}_{1}\right.$ is true $)$ and in red, values obtained for different ink samples were compared $\left(\mathrm{H}_{2}\right.$ is true). The horizontal bars in the boxplots represent the median, the box all values between 25 and $75 \%$ percentile, and the whiskers at 1.5 times the interquartile range. Outliers are represented by crosses. 